\title{
Chicken Bursal Lymphoma
}

National Cancer Institute

\section{Source}

National Cancer Institute. Chicken Bursal Lymphoma. NCI Thesaurus. Code C134556.

A malignant lymphoma that arises in the bursa of Fabricius in a chicken. 